\title{
INFLUENCE OF SOLAR LUMINOSITY OVER GEOMAGNETIC AND CLIMATIC CYCLES AS DERIVED FROM SPELEOTHEMS
}

\author{
Y. Shopov, D. Stoykova, L. Tsankov, M. Sanabria, D. Georgieva, D. Ford, L. Georgiev
}

\begin{abstract}
We observed cycles presented in a luminescent solar insolation proxy record from a speleothem from Jewel Cave, South Dakota, US. We found cycles of orbital precession with periods of 23 and $19 \mathrm{ka}$ and of obliquity of $41 \mathrm{ka}$ and many others from non- orbital origin in this sample. We determined the Solar origin of the cycles with durations of $11500,4400,3950,2770,2500,2090,1960,1670,1460,1280,1195,1145,1034,935$, 835,750 and 610 years. It was done by their detection both in proxy records of speleothem luminescence, $\Delta{ }^{14} \mathrm{C}$ and the intensity of the geomagnetic dipole. It is well known that the main variations in the last two records are produced by the solar wind. The most intensive cycle discovered in this record has duration of $11.5 \mathrm{ka}$. It is not of orbital origin. It was found previously to be the most intensive cycle in the $\Delta^{14} \mathrm{C}$ calibration record and has been interpreted to be of terrestrial origin because "it is too strong to be of solar origin". Our studies suggest that it should be a solar cycle modulating the geomagnetic field and ${ }^{14} \mathrm{C}$ reversed production as the other solar cycles do.
\end{abstract}

Keywords: luminescence, ${ }^{14} \mathrm{C}$ - dating, geomagnetism, solar luminosity.

\section{Milankovitch Theory and Experimental records}

According to the astronomical theory of paleoclimate (Milankovitch theory) the longterm variations in the geometry of the Earth's orbit are the fundamental causes of Pleistocene Ice Ages. The orbital elements that cause variations in solar insolation are:

1. Eccentricity - the ratio between the distance from the centre of the orbit to one of its focal points versus the semi-major axis of the Earth's orbit. It varies with several periods with duration around 100,000 years. They cause small variations of the total solar energy received by the Earth.

2. Variations of the tilt (obliquity) of the Earth's axis with respect to the vertical to the ecliptic plane (the plane of the Earth's orbit). It is calculated to change about $\pm 1.5^{\circ}$ on either side of its average angle of $23.5^{\circ}$, with a period of about 41,000 years.

3. Precession - owing to the gravitational pull of the Sun and the Moon on the equatorial bulge of the Earth, its axis of rotation moves slowly around a circular path and completes one turn in about 21,000 years: more precisely, it has two major modes at 23,000 and 19,000 yrs respectively. It causes also precession of the equinoxes and the solstices with the same period. Precessional motion is similar to rotation of the axis of a spinning top. These orbital and insolation changes have been calculated theoretically for the past several millions years (Berger et al., 1991). Development of paleoclimate dating methods made possible testing of the theory by comparison of the predicted patterns of climate 
change with experimental proxy paleoclimate records from the ocean, lake and ice cores, speleothems, etc. According to the theoretical calculation of Berger (1989) the influence of the eccentricity on the total insolation received by the Earth is quite small - only $0.1 \%$ of it or less. Thus insolation spectra should be dominated by the precession and obliquity but in fact the strongest cycle has duration of about 100,000 yrs in experimental records and obliquity dominates over the precession variations. Theoretical orbital variation curves explain about $1 / 2$ of the variation in the existing proxy paleotemperature records (Imbrie et al.,1992; 1993) derived from sea cores and polar ice. Precise correlation demonstrates that a significant part of the signal is not due to the components of the orbital variations (Imbrie et al., 1992; 1993) in the proportions suggested by the theoretical solar insolation curves. In order to overcome this disagreement Imbrie (1985) introduced his ETP index of orbital variations, which is a sum of the separate components of orbital variations in proportions other than those suggested by Milankovitch theory. Although ETP curves correlate far better with experimental paleoclimatic curves, introduction of the ETP index does not have any physical basis. Imbrie (1985) does not discuss why the real ratio between orbital variations differs from the theoretical one.

Solar variations may drive glaciers melting as suggested by (Shopov, 2002). Rapid temperature changes such as the well known "Little Ice Age" and "Medieval Maximum" of historical times are often connected with changes of the solar activity. Understanding of the processes leading to formation and melting of the ice sheets is of great significance for prediction of possible climate disasters in the future.

\section{Results and Discussion}

We measured a luminescent solar insolation proxy record in a speleothem (JC11) from Jewel Cave, South Dakota, US (Shopov et al., 2002). It covers the period from 89,300138,600 yrs B.P. with high resolution (34 years) and precision of measurements better than $1 \%$. Here we publish the TIMS U/Th dates of this record (Table 1). Their 2 - sigma error varies from 0.8 to 5.5 kyrs.

Table 1. TIMS U/Th dates of a speleothem JC 11

\begin{tabular}{ccccc}
\hline Sample N & $\begin{array}{c}\text { Distance from the top } \\
(\mathbf{m m})\end{array}$ & 230Th/ 234U & $\begin{array}{c}\text { 230Th/ 234U Age } \\
\left(10^{3} \text { years }\right)\end{array}$ & $\begin{array}{c}2 \sigma \text { error } \\
\left(10^{3} \text { years }\right)\end{array}$ \\
\hline B10 & $0-5$ & 0.568 & 89 & 8.0 \\
WXL-1 & $2-3$ & & 92 & 0.8 \\
JC11-1 & $3-6$ & 0.6046 & 97.5 & 2.5 \\
JC11-1B & $8-10$ & 0.6415 & 107 & 2.3 \\
B9-10 & $10-15$ & 0.664 & 113 & 13.0 \\
JC11-2 & 17 & 0.7243 & 133 & 5.5 \\
\hline
\end{tabular}

We designed a special algorithm ("Real Space” periodogramme analysis) and supporting computer program to compare quantitatively the intensities of all cycles present in our data. It plots the periodogramme in coordinates of the real space- Cycle Intensity/Period (in years).

We extracted orbital variations from the JC11 record by a band-pass Tukey filter set for 
frequencies of 41, 23 and 19 kyrs (Fig.1). Intensity of the component of the solar insolation variations due to tilt appears to be twice more intensive than that due to the precession variations. This result contradicts the suggestion of the orbital theory that precession variations should be responsible for almost the entire solar insolation variation for this place and time period (Berger et al., 1991).

There are two possible explanations of this disagreement:

1. Variations of the solar insolation caused by the precession are far less than those suggested by theory.- This is very unlikely.

2. The amplitude of obliquity variations was greater than the ones predicted by the theory.-Such greater variations are possible. It may be caused by redistribution of the ocean masses as a result of variations of the obliquity. Variations of the inertial moment of the Earth (due to the Sea level changes caused by formation or melting of ice sheets during glacial periods) produce variations of the rotation speed and oblateness of the Earth. These processes may also lead to variations of the obliquity. Orbital theory (Berger et al., 1991) did not take into account such variations of the physical properties of the Earth. Tenchov and Tenchov (1993) demonstrated that the formation of ice sheets change the rotation speed and in result the oblateness of our planet. Although quantitative assessment of the influence of glaciations on these physical properties and resulting effects on Earth's orbit still needs further work.

Sonett (1984) found that a cycle with a period of about 900 yrs has intensity 5-7 times higher than that of the well- known centennial cycle in the ${ }^{14} \mathrm{C}$ solar proxy record. Stuiver and Braziunas (1989) calculated MEM- spectra of the same record and claimed that "changes occur in the Sun' s convective zone with a fundamental oscillatory mode of about $420 \mathrm{yr}$ period." and that century and sub-century cycles are about one twentieth of the strength of this $420-y r$ cycle. Although the uncertainty of the proportion between intensities of different cycles in the spectra calculated by these authors cannot be estimated they suggest that longer solar cycles are more than one order of magnitude stronger than the solar cycles detected by direct measurements of sunspot number, the Solar Constant, etc.

Periodogrammes we calculated from the JC11 luminescence record demonstrate that the 900 year solar cycle has an intensity only $3-4 \%$ of the $11,500-y r$ cycle and the solar cycle of about 420 years has intensity less than $2.5 \%$ of the 11,500 -yr cycle. The $11,500-$ yr cycle thus should have an intensity that is several orders of magnitude higher than the century and sub-century cycles (which are measured directly). We found many other cycles (Table 2), most intensive of them with amplitude ranging respectively from 7.3 to $0.7 \%$ of the Solar Constant. All these cycles are statistically significant and well above the experimental error.

Berger et al. (1991) calculated the theoretical intensity of the precession cycle in $\mathrm{W} / \mathrm{m}^{2}$ and $\%$ of Solar Constant. We used these calculations to calibrate our record in $\mathrm{W} / \mathrm{m}^{2}$ and in $\%$ of Solar Constant.

We used the same computer program to detect a cycle with duration of $10,026(+1254 /$ 834) yrs in an independent record of the intensity of the geomagnetic field of the Earth (Tric et al., 1992). We correlated this cycle with the 11,500 (+1900/-1150) yrs cycle in the luminescent record and then calibrated all other cycles in the geomagnetic record to this luminescent cycle. This cycles coincides in the frames of experimental error of determination of 


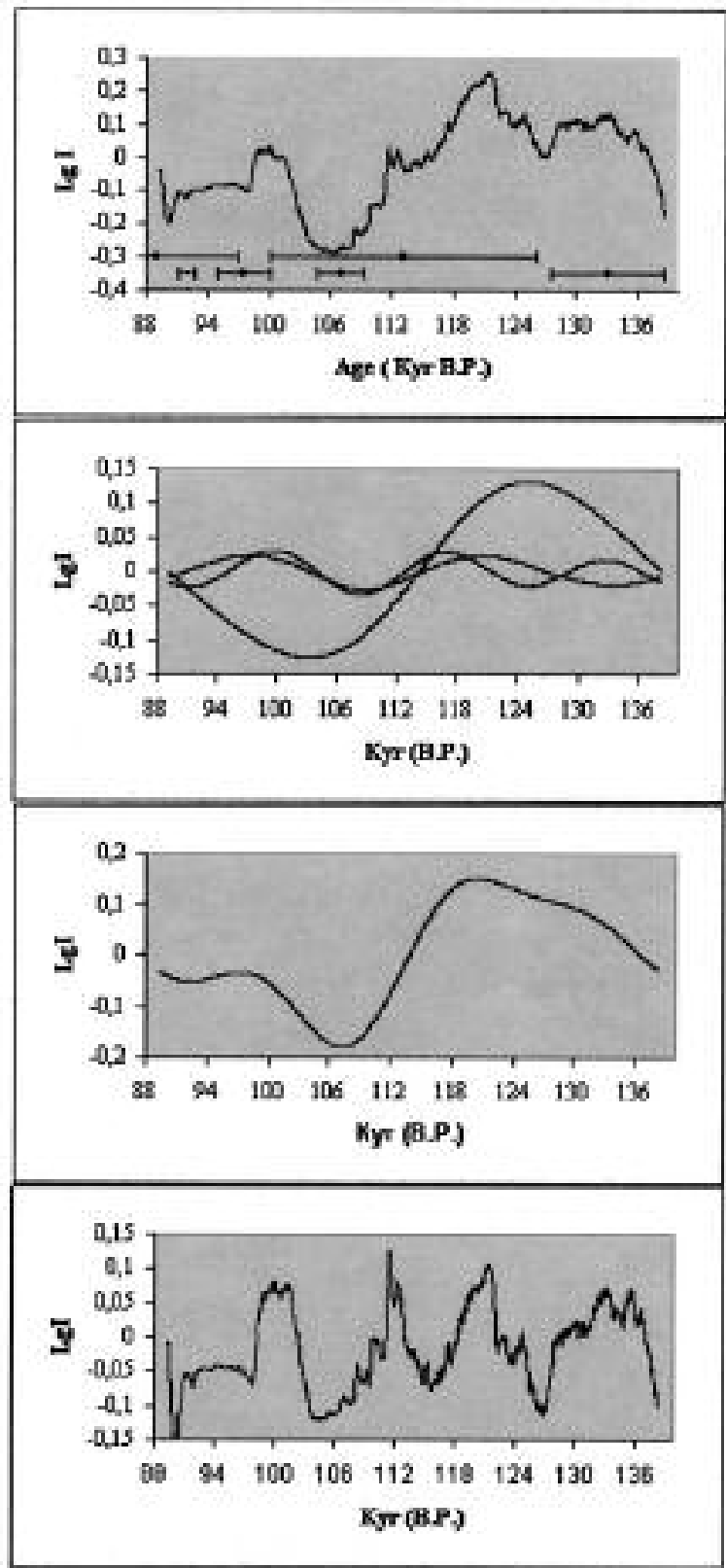

Figure 1. (a) Jewel Cave (South Dakota) Speleothem JC11 luminescence record (Shopov et al. 2002). The TIMS U/Th dating points are shown by points with $2 \sigma$ error bars. (b) Orbital components of Solar Insolation variations extracted from the record of Fig. 1(a) by a band-pass Tukey filtration set for the frequencies of 41,23 and 19 kyrs of the orbital cycles, (c) sum of the orbital variations (from Fig. 1(b)), (d) Solar Luminosity variations extracted from Solar Insolation proxy record (a) by subtracting of the sum of the orbital variations (c) from the JC 11 luminescent record. All curves are presented in logarithm of intensity of luminescence (|gl). 
its duration. It was found that many identical cycles appear in both records (Tab. 2).

Solar cycles modulate both reversed production of cosmogenic isotopes such as ${ }^{14} \mathrm{C}$, ${ }^{10} \mathrm{Be}$, etc (Sonett 1984, Stuiver \& Braziunas, 1989) and geomagnetic field intensity (Tric et al., 1992) through solar wind. At the same time climatic cycles are not able to modulate cosmogenic isotopes reversed production and especially geomagnetic field intensity. So if one cycle is presented both in a paleoclimatic proxy and in proxy records of the geomagnetic field intensity or of cosmogenic isotopes reversed production it definitely determines solar origin of the cycle in the paleoclimatic record. This demonstrates the solar origin of the cycles with durations of 11500, 4400, 3950, 2770, 2500, 2090, 1960, 1670, 1460, 1280, $1195,1145,1034,935,835,750$ and 610 years (Tab. 2). The rest of the cycles presented in the luminescent record may still be of solar origin, but they could not be detected in the ${ }^{14} \mathrm{C}$ and geomagnetic field intensity records due to the lower resolution of their periodogrammes. This is result of the lower resolution of the last two records and their shorter duration, so cannot be overcome with the available records of these solar proxies. At the same time periodograme of the luminescence record have several times higher resolution.

\begin{tabular}{|c|c|c|c|c|}
\hline Cycle (Yrs) & $\operatorname{VSC}\left(\mathrm{W} / \mathrm{m}^{2}\right)$ & PSC (\%) & GEOM (\%) & ${ }^{14} \mathrm{C}(\%)$ \\
\hline 11500 & 100.6 & 7.33 & 7.33 & 7.33 \\
\hline 7800 & 27.5 & 2.00 & & \\
\hline 6160 & 41.5 & 3.02 & & \\
\hline 4400 & 24.4 & 1.78 & 6.2 & \\
\hline 3950 & 25.1 & 1.83 & 4.32 & \\
\hline 3400 & 24.1 & 1.76 & & \\
\hline 2770 & 12.6 & 0.92 & 3.25 & \\
\hline 2500 & 16.3 & 1.19 & 4.70 & \\
\hline 2300 & 12.5 & 0.91 & & \\
\hline 2090 & 7.28 & 0.53 & 2.26 & \\
\hline 1960 & 11.26 & 0.82 & 2.82 & \\
\hline 1770 & 8.5 & 0.62 & & \\
\hline 1670 & 9.1 & 0.66 & 1.01 & 0.73 \\
\hline 1460 & 10.0 & 0.73 & 2.42 & \\
\hline 1280 & 4.8 & 0.35 & 0.71 & \\
\hline 1195 & 4.5 & 0.33 & 1.22 & 0.40 \\
\hline 1145 & 4.5 & 0.33 & 1.92 & \\
\hline 1034 & 4.26 & 0.31 & 1.62 & \\
\hline 935 & 3.02 & 0.22 & 1.2 & \\
\hline 835 & 3.6 & 0.26 & 0.6 & 0.56 \\
\hline 814 & 2.6 & 0.19 & & \\
\hline 775 & 2.3 & 0.17 & & \\
\hline 750 & 2.6 & 0.19 & & 0.56 \\
\hline 670 & 2.06 & 0.15 & & \\
\hline 660 & 2.47 & 0.18 & & \\
\hline 610 & 1.78 & 0.13 & & 0.48 \\
\hline 570 & 2.06 & 0.15 & & \\
\hline 550 & 1.78 & 0.13 & & \\
\hline 538 & 2.06 & 0.15 & & \\
\hline
\end{tabular}

Table 2. Cycles of Solar Luminosity (in Years) that have been detected in the speleothem record, calculated variations of the Solar Constant expressed in W/m² (VSC) and in \% from the Solar Constant (PSC), compared to cycles in two Solar Wind proxies- the Intensity of the Dipole of the Geomagnetic Field (GEOM) and the reversed rate of production of ${ }^{14} \mathrm{C}$ in the upper atmosphere. All cycles listed here are calculate with the same computer program to produce comparable cycles. 


\section{Conclusions}

We determined the Solar origin of the cycles with durations of 11500, 4400, 3950, 2770, $2500,2090,1960,1670,1460,1280,1195,1145,1034,935,835,750$ and 610 years. It was done by their detection both in proxy records of speleothem luminescence, ${ }^{14} \mathrm{C}$ and the intensity of the geomagnetic dipole. These solar cycles modulate the geomagnetic field and the reversed production of cosmogenic isotopes producing their variations with the same duration.

Solar luminosity has very strong variations. It has most prominent cycle of 11,500 yrs and a number of shorter cycles. All of them are able to affect the climate of the Earth. Speleothem records, as the best dated paleoclimatic records over the past 500 ka may serve as reliable sources for proxy records of the solar insolation and solar luminosity.

\section{Acknowledgements}

This research have been funded by Bulgarian Science Foundation by research grant $811 / 98$ to Y. Shopov

\section{References}

Berger A., 1989 - Pleistocene Climatic Variability at Astronomical Frequencies. Quat. Int., 2: 1-14.

Berger A., Loutre M.F., Laskar J., 1991 - Insolation Values for the Climate of the last 10 Million Years. Quat. Sci. Res., 10: 297-317.

Imbrie J., 1985 - A Theoretical Framework for the Pleistocene Ice Ages. J. Geol. Soc. London, 142: 417432.

Imbrie J., Boyle E.A., Clemens S.C., Duffy A., Howard W.R., Kukla G., Kutzbach J., Martinson D., Mix A.C., Molfino B., Morley J., Peterson L.C., Pisias N.G., Prell W.L., Raymo M.E., Shackleton N.J., Toggweiler J.R., 1992 - On the Structure and Origin of Major Glaciation Cycles 1. Linear Responses to Milankovich Forcing. Paleoceanography, 7(6): 701-736.

Imbrie J, Boyle E.A., Clemens S.C., Duffy A., Howard W.R., Kukla G., Kutzbach J., Martinson D., Mix A.C.,

Molfino B., Morley J., Peterson L.C., Pisias N.G., Prell W.L., Raymo M.E., Shackleton N.J., Toggweiler J.R., 1993 - On the Structure and Origin of Major Glaciation Cycles 2. The 100,000-year Cycle. Paleoceanography, 8(6): 669- 735.

Shopov Y.Y., Stoykova D. A., Tsankov L., Sanabria M., Lundberg J., Georgiev L.N., Forti P., Georgieva D., 2002 - Verification of the Causes of Glaciations and Sea Level Changes Using the Records of Calcite Speleothems. International Journal of Speleology, 29 B(1/4): 71-75.

Sonett C.P., 1984 - Very Long Solar Period and the Radiocarbon Record. Review of Geophysics and Space Physics, 22(3): 239-254.

Stuiver M., Braziunas T., 1989 - Atmospheric ${ }^{14} \mathrm{C}$ and Century- Scale Solar Oscillations. Nature, 3: 405407.

Tenchov G. G., Tenchov Y. G., 1993 - An Estimation of Geological Factors Affecting the Long Time Earth Spin Rotation. Compt. Rend. I'Acad. Bulg Sci., 46(12): 37-40.

Tric E., Valet J-P., Tucholka P., Paterne M., Labeyrie L., Guichard F., Fotugne M., 1992 - Paleointensity of Geomagnetic Field During the Last 80,000 Years. J Geophys. Res, 97: 9337-9351. 